\title{
Identification of a Novel Mutation (p.G328W) in the NR5A1 Gene in a Boy with 46, XY DSD: Case Report of Clinical, Endocrine and Genetic Features
}

\author{
Ortolano $\mathrm{R}^{1 *}$, Baldazzi $\mathrm{L}^{1}$, Menabò $\mathrm{S}^{2}$, Balsamo $\mathrm{A}^{3}$, Cassio $\mathrm{A}^{2}$, Antona $\mathrm{V}^{4}$, Cimador $\mathrm{M}^{4}$, Corsello $\mathrm{G}^{4}$ and Giuffrè $\mathrm{M}^{4}$ \\ ${ }^{1}$ Department of Woman, Child Health and Urologic Diseases, Pediatric Unit, S.Orsola-Malpighi University Hospital, Italy \\ ${ }^{2}$ Department of Medical and Surgical Sciences, Pediatric Unit, University of Bologna, Italy \\ ${ }^{3}$ Center for Rare Endocrinologic Conditions (CARENDO BO; Endo-ERN), S.Orsola-Malpighi University Hospital, Bologna \\ ${ }^{4}$ Department of Sciences for Health Promotion and Mother and Child Care G. D'Alessandro, Italy
}

*Corresponding author: Ortolano R, Department of Woman, Child Health and Urologic Diseases, Pediatric Unit, S.Orsola-Malpighi University Hospital, Via Massarenti 11, 40138 Bologna, Italy

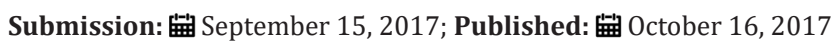

\section{Abstract}

Introduction: The gene NR5A1 (nuclear receptor subfamily 5 group A member 1, OMIM +184757) encodes for the Steroidogenic factor1 (SF1), a key regulator of the adrenal/gonadal development and function. Disorders/Differences of Sex Development (DSD) are defined as conditions where the chromosomal/anatomical, phenotypic sex is atypical. Heterozygous mutations of NR5A1 gene are associated, in 46, XY DSD patients, with a wide phenotypic spectrum of the external genitalia, with or without adrenal failure. Here we report a 46, XY newborn carrying a novel heterozygous NR5A1 mutation that presents with ambiguous genitalia without adrenal failure.

Case Presentation: newborn with ambiguous genitalia at birth (micropenis, hypospadias) with normal testosterone (T) response to hCG test, with a Müllerian remnant and bilateral inguinal immature gonads. Molecular analyses for AR, SRD5A2 genes did not reveal any mutation. NR5A1 sequence analysis identified a novel substitution c.982G>T (GGG>TGG) in exon 5, which results in the non conservative p.G328W mutation, that arises de novo. Evaluation of the adrenal function was normal and no symptoms or signs of adrenal insufficiency have emerged during follow-up.

Conclusion: This is the first description of the p.G328W mutation (Sequence reference NC_000023.10) of the NR5A1 gene that causes the replacement of a highly conserved residue of the LBD (ligand binding domain) and very likely affects the SF1 action. This finding contributes to choose sex assignment and underlines the importance to perform the analysis of the NR5A1 gene also in 46, XY DSD patients with a PAIS-like phenotype.

Keywords: Disorders of sex development; Differences of sex development; DSD, NR5A1 gene; Steroidogenic factor-1; Ambiguous genitalia

Abbreviations: ACTH: adrenocorticotropic hormone; $\triangle 4 \mathrm{~A}: \triangle 4$ Androstenedione; DHEAS: dehydroepiandrosterone sulphate; DHT: dihydrotestosterone; DSD: Disorders or Differences of Sex Development; HCG: Human Chorionic Gonadotropin; MRI: magnetic resonance imaging; PAIS: Partial Androgen Insensitivity Syndrome; SPL: Stretched Penile Length; T: Testosterone

\section{Introduction}

Disorders of Sex Development (DSD) comprise a variety of conditions where the chromosomal, gonadal or phenotypic sex are atypical [1]. Because of a diffused negative connotation of the term "disorders" perceived by some advocacy organizations, recently it has been proposed to prefer the word "differences" [2]. 46, XY DSD, in particular, consist of a wide range of phenotypes, from very low under virilization through various degrees of ambiguity, to complete female appearance. They are caused by partial or total gonadal dysgenesis, defects of androgen synthesis/action or other causes. To date, only the $50 \%$ of the cases receive a correct diagnosis, including the identification of the genetic/hormonal cause [3].

In the last 10 years, the importance of the Steroidogenic factor 1 (SF1) [4] as a crucial factor has increased and its implication in a wide spectrum of DSD phenotypes has emerged [5]. SF1 is one of the most precocious markers of the adrenal/gonadal development, initially identified as an orphan nuclear receptor. Key determinant of the tissue-specific expression of steroidogenic enzymes it binds a wide range of different phospholipid and sphingolipid ligands [6,7]. In mice and humans it is expressed very early in the urogenital ridge and continues to be highly expressed in the developing adrenal, gonad, ventromedial hypothalamus, and pituitary.

In male SF1 is necessary for testis determination and differentiation: for the formation of bipotential gonads, to activate the expression of anti-Müllerian hormone gene (AMH) in Sertoli cells [8] and to regulate the expression of steroid hormones in the Leydig cells. In female development, it is also actively present and participates in different steps of ovarian development and function: it is expressed in the granulosa and theca cells where it regulates 
genes required for ovarian steroidogenesis and follicle growth maturation [9]. SF1 has 461 amino acids and is encoded by the gene NR5A1 (nuclear receptor subfamily 5 group A member 1, OMIM + 184757) located at 9q33 and consisting of 7exons.

The SF1 structure typically includes: a DNA binding domain (DBD) with two zinc fingers, a hinge region and a ligand-binding domain (LBD) [10]. NR5A1 mutations in 46,XY subjects may lead to various degree of undervirilization: first described in patients with Müllerian remnants and primary adrenal failure [11], subsequently over 50 mutations were reported mainly in 46,XY DSD individuals/ infertile males, sometimes also resembling a PAIS/MAIS phenotype, with apparently normal adrenal function [12,13]. 46, XX females with NR5A1 mutations may present with or without primary adrenal and or ovarian insufficiency $[14,15]$. The NR5A1 mutations are usually present in heterozygous condition and very rarely in homozygosity, thus suggesting a potential dosage-dependent action of SF1 [16]. Different penetrance and variable inheritance pattern for NR5A1 mutations were also well documented among various families, thus indicating a complex phenotype expressivity [3]. This wide range of expressions enlarges the difficulties to establish a direct genotype-phenotype correlation [17] and hampers both the genetic counselling and the decisions making for management at the time of the diagnosis [2].

Any novel findings that enlarge the spectrum of cases is therefore helpful to improve the comprehension of the genotype-phenotype correlation. Here, we report a novel heterozygous substitution c.982G $>$ T (GGG>TGG) in exon 5, which results in the p.G328W mutation, de novo, identified in a 46, XY DSD patient with severe undervirilization without adrenal insufficiency.

\section{Case Presentation}

The study was undertaken under an institutionally approved ethic protocol and informed consent was obtained from relatives. The patient was born at term by third pregnancy to healthy nonconsanguineous parents. At birth the neonate presented with ambiguous genitalia: micropenis, single perineal urogenital opening representing a urogenital sinus [2], mild hyperpigmentation of labioscrotal folds without palpable gonads registered as female. Cytogenetic analysis revealed a 46, XY karyotype; therefore the infant was referred to our service at 20 days of life for 46, XY DSD. Physical examination revealed a stretched penile length (SPL) of $1.5 \mathrm{~cm}$, a single perineal urogenital opening, and palpable gonads in the labioscrotal folds (Figure $1 \mathrm{a}-\mathrm{b}$ ). The external masculization score was 5 .

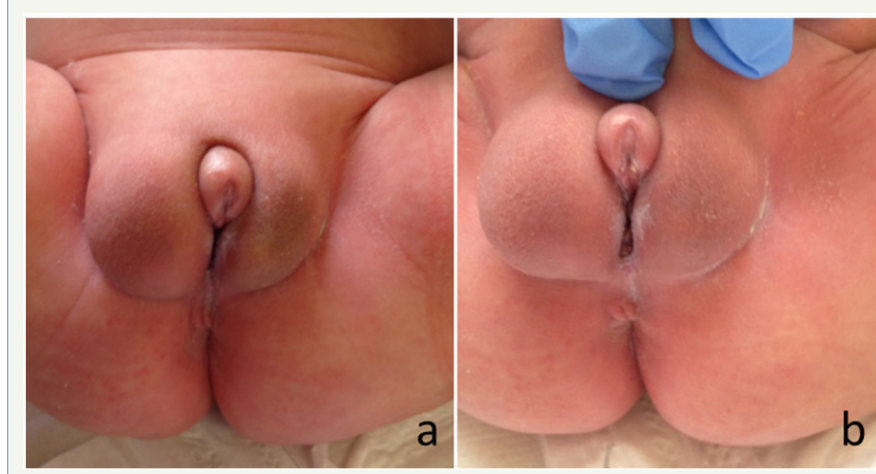

Figure1(a-b): External genitalia at 1 month of age: $1.5 \mathrm{~cm}$ phallus, single perineal opening and palpable gonads in the labioscrotal folds.

Hormonal investigations showed a normal testosterone (T) response to hCG test (Table 1 ), a basal T/ $\Delta 4$ Androstenedione (T/ $\Delta 4 \mathrm{~A}$ ) ratio of 7,5 , a basal $\mathrm{T} /$ dihydrotestosterone (T/DHT) ratio of 9 , 5. Evaluation of the adrenal function indicated that basal cortisol (F) and dehydroepiandrosterone sulphate (DHEAS) were within normal ranges (Table 2) and no symptoms or signs of adrenal insufficiency have emerged during follow-up. Pelvic ultrasound showed testicular-like structures inside labioscrotal folds and a Müllerian remnant confirmed by pelvic magnetic resonance imaging (MRI) and by exploratory laparoscopy. Genitography disclosed a structure similar to a closed-end vagina.

Table 1: Laboratory response to hCG test at 3 months of life (1000 UI/day of hCG for 3 consecutive days).

\begin{tabular}{|c|c|c|c|c|c|c|c|c|}
\hline & $\begin{array}{l}\text { FSH } \\
\text { mUI/mL }\end{array}$ & $\begin{array}{l}\text { LH } \\
\text { mUI/mL }\end{array}$ & $\begin{array}{l}\text { E2 } \\
\mathrm{pg} / \mathrm{mL}\end{array}$ & $\begin{array}{l}\mathrm{P} \\
\mathrm{ng} / \mathrm{mL}\end{array}$ & $\begin{array}{l}\mathrm{T} \\
\mathrm{ng} / \mathrm{ml}\end{array}$ & $\begin{array}{l}\Delta 4-\mathrm{A} \\
\mathrm{ng} / \mathrm{mL}\end{array}$ & $\begin{array}{l}\text { DHT } \\
\mathrm{ng} / \mathrm{mL}\end{array}$ & $\begin{array}{l}\text { DHEAS } \\
\mu \mathrm{g} / \mathrm{dL}\end{array}$ \\
\hline T0 & 5.6 & 3.69 & $<5$ & 0.167 & 1.5 & 0.2 & 0.158 & 6.81 \\
\hline $\mathrm{T} 4$ & 2.27 & 0.32 & $<5$ & 0.03 & 4.3 & 0.14 & NA & NA \\
\hline
\end{tabular}

$\mathrm{TO}=$ basal value; $\mathrm{T} 4=$ values at day 4 after $\mathrm{hCG}$ administration; $\mathrm{E} 2=$ estradiol; $\mathrm{P}=$ progesterone.

NA=not avaible

Table 2: Evaluation of gonadal and adrenal basal function.

\begin{tabular}{|l|l|l|l|l|l|l|l|l|l|l|}
\hline $\begin{array}{l}\text { Age } \\
\text { Months }\end{array}$ & $\begin{array}{l}\text { FSH } \\
\mathbf{m U I} / \mathbf{m L}\end{array}$ & $\begin{array}{l}\mathbf{L H} \\
\mathbf{m U I} / \mathbf{m L}\end{array}$ & $\begin{array}{l}\mathbf{E 2} \\
\mathbf{p g} / \mathbf{m L}\end{array}$ & $\begin{array}{l}\mathbf{T} \\
\mathbf{n g} / \mathbf{m L}\end{array}$ & $\begin{array}{l}\mathbf{P} \mathbf{n g} / \mathbf{m L} \\
\mathbf{m g} / \mathbf{d L}\end{array}$ & $\begin{array}{l}\mathbf{F} \text { at 8:00 AM } \\
\mathbf{m g} / \mathbf{d L}\end{array}$ & $\begin{array}{l}\mathbf{F} \text { at 8:00 PM } \\
\mathbf{m g} / \mathbf{d L}\end{array}$ & $\begin{array}{l}\text { ACTH } \\
\mathbf{p g} / \mathbf{m L}\end{array}$ & $\begin{array}{l}\text { Renin } \\
\mathbf{n g} / \\
\mathbf{m L} / \mathbf{h}\end{array}$ \\
\hline 2 & 7.41 & 3.72 & $<5$ & 2.03 & 0.145 & 21.03 & $\mathrm{NA}$ & $\mathrm{NA}$ & $\mathrm{NA}$ & $\mathrm{NA}$ \\
\hline 3 & 4.9 & 1.74 & $<5$ & 0.817 & 0.134 & 24.1 & 17.13 & 1.93 & 67.4 & 2.51 \\
\hline
\end{tabular}

$\mathrm{NA}=$ not available; $\mathrm{F}=$ cortisol; 
Bilateral gonadal biopsy was performed at 4 months of age and the histological investigation showed: on the right gonad, immature testis with seminiferous tubules with Sertoli cells and only few germ cells; on the left gonad testicular parenchyma with seminiferous tubules containing few germ cells at the basal lamina. A partial gonadal dysgenesis, as suggested by the presence of a Müllerian remnant, cannot be excluded, probably more evident at the gonadal poles not included in the biopsy. No ovarian-like stroma was observed and OCT3/4 and CD117 immuno-hystochemical markers were negative on both gonads.

\section{Methods}

Genital ambiguity was evaluated according to Ahmed et al. [18]. SPL is determined by measuring the distance from the base of the penis under the pubic symphysis to the tip of the glans [19]. Blood samples were drawn in the morning (08:00-09:30 a.m.) Serum levels of LH, FSH, $\triangle 4 \mathrm{~A}$, T, DHT, cortisol and ACTH were measured by commercial kits. The human Chorionic Gonadotropin (hCG) stimulation test was performed with recombinant hCG Gonasi (C) 1000 UI/day of hCG for 3 consecutive days. Genomic DNAs from patient, parents and 2 sisters were purified from peripheral leukocytes by MagNA Pure Compact system (Roche) and analysed by means of PCR and Sanger sequencing of the coding and splicing regions of the genes: $S R Y, A R, S R D 5 A 2$ and NR5A1 (list of primers/ PCR conditions will be provided under request).

\section{Results}

Sanger sequencing of the genes $S R Y, A R, S R D 5 A 2$ did not show any mutation. The analysis of NR5A1 gene showed an heterozygous substitution c.982G>T (GGG>TGG) in exon 5, which results in the substitution of Glycine with Tryptophan at position 328 (reference Sequence NC_000023.10): p.G328W; the result was confirmed in two independent PCR/sequencing reactions on both filaments (Figure 2a). Molecular investigation of the family members was negative in all of them, thus this novel variant has originated de novo. This novel non-synonymous mutation affects the highly conserved Gly328, located on one of the 2 beta sheets between helix 5 and helix 6 of the LBD (Figure 2b).

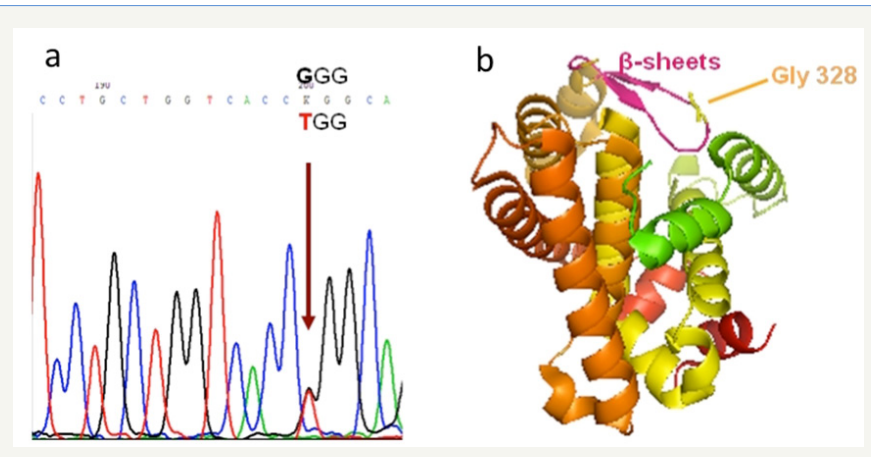

Figure2a: Electropherogram of the identified mutation: the arrow points to the heterozygous substitution $G>T$ at codon 328 (GGG>TGG).

Figure2b: 3D cartoon of the Ligand Binding Domain of the SF1 protein: in magenta the 2 beta sheets between helix $5-6$ and in yellow the Gly 328 residue.

\section{Discussion}

We present here an Italian case of 46, XY DSD with ambiguous genitalia at birth that harbors a novel NR5A1 mutation, without other mutations affecting $A R \& S R D 5 A 2$ genes. Patient phenotype was compatible with SF1 deficit Type II according Achermann's classification [12]. He presents a normal male testosterone production after hCG test with a partial gonadal dysgenesis suggested by the presence of a Müllerian remnant. This mutation, p.G328W, originates de novo and affects a highly conserved and small residue of one of the $2 \beta$ sheets. Taken together the degree of conservation, the type of substitution (different characteristics between the small Gly compared to the cumbersome Trp) and the de novo origin it is likely to speculate that this mutation is involved in the observed phenotype and functional studies of the mutant are in progress.

A different missense mutation affecting the same residue (p.G328V) was recently described in a case $46, \mathrm{XY}$ with severe penoscrotal hypospadias, micropenis, and small inguinal testis without adrenal failure [20]. This patient was assigned male sex and showed spontaneous virilization and male pubertal development but subsequently azoospermia. Functional analysis of the mutant showed a reduction to $8 \%$ of wild-type activity on the Cyp 11 a promotor in vitro, thus supporting the hypothesis of an involvement of p.G328W in the observed phenotype, also considering that Valine is much more similar to Glycine than Tryptophan.

The sex initially assigned was female, then changed in male after the hormonal, genetic and psychological evaluations. He was treated with intramuscular administration of $25 \mathrm{mg}$ testosterone enanthate once a month for 3 months [19] with an increase in his penile growth (SPL $3 \mathrm{~cm})$.

Surgery was programmed to descend testes and to correct urethral opening. There are reports of $46, \mathrm{XY}$ DSD patients due to heterozygous mutations in the NR5A1 gene with male sex reassignment, orchidopexy and removal of Müllerian remnant [21]. Some studies [20,22] showed that patients with NR5A1 mutations could produce sufficient levels of testosterone to enter puberty spontaneously but with potential progressive gonadal dysgenesis resulting in oligo-azoospermia. Therefore it is recommended a regular monitoring of gonadal function in adolescence and adulthood, with eventual early cryopreservation of sperms. The risk for tumor development in patients with NR5A1 mutations is intrinsically increased due to testicular dysgenesis.

The risk is major on dysgenetic and undescended gonads [5] for the prolonged non-scrotal location of the gonads that has been recognized as an additional risk factor [23]. Our patient presents a gonadal histology characterized by some Sertoli cells and few spermatogonia and Leydig cells as described in other patients with SF1 deficit reported in literature $[24,25]$ and with OCT3/4 negative that should indicate a lower tumor risk. In contrast to the relatively small number of $N R 5 A 1$ changes reported in association with adrenal failure, heterozygous NR5A1 changes are emerging as a relatively frequent finding in patients with $46, \mathrm{XY}$ DSD without adrenal insufficiency [26]. It has been proposed that genetic events 
leading to haploinsufficiency of NR5A1 could disrupt testicular development and function, whilst adrenal function remained intact [5].

Evaluation of the adrenal function in our patient showed normal basal cortisol, DHEAS and ACTH without any signs or symptoms of adrenal insufficiency but he will have to perform an ACTH test and follow-up for adrenal function.

\section{Conclusion}

Heterozygous NR5A1 changes are emerging as a relatively frequent finding in patients with 46, XY DSD without adrenal failure [12], like our patient. In some individuals sex assignment is challenging and long-term studies of gender identity and psychosexual functioning are needed [27]. We report a novel mutation of NR5A1 involving a novel substitution c.982G>T (GGG>TGG) with glycine replacing tryptophan in exon 5 presenting with ambiguous genitalia without adrenal failure. It is the first description of the p.G328W mutation of the NR5A1 gene, which causes replacement of a highly conserved residue of the ligand binding domain and likely affects SF1 action. This finding helps in choosing sex assignment and underlines the importance of performing the analysis of NR5A1 gene also in 46, XY DSD patients with a PAIS-like orphan phenotype.

The long-term follow-up will supply useful information to understand if the choice of male sex of rearing has been appropriate. More studies are necessary to understand the phenotype/genotype correlation and to predict spontaneous pubertal development and potential future fertility.

\section{Ethics Approval and Consent to Participate}

The phenotypic data was collected from routine clinical examination and clinical records. Informed written consent for every genetical investigation performed to identify the underlying cause of DSD was obtained in accordance with protocols that comply with the Declaration of Helsinki. Therefore, no ethical approval was necessary.

\section{Consent to Publish}

Written informed consent for publication has been obtained by patient's parents.

\section{Availability of Data and Materials}

DNA samples and biochemical results are stored in the Genetic Laboratory of Bologna and The official clinical chart of Palermo Hospital, respectively and will be available on specific request.

\section{Authors ‘ Contributions}

RO was responsible for the clinical and endocrine evaluation of the patient. BL performed genetic counselling and conducted diagnosis with RO. SM and LB performed the molecular genetics studies of the genes AR, SRD5A2 and NR5A1. RO, BL, AB, AC, MG, GC participated in the design of the study. MC was responsible for laparoscopic and biopsy investigations. RO, BL, MG conceived the study, and participated in its design and coordination and helped to draft the manuscript. All authors read and approved the final manuscript.

\section{Acknowledgement}

We wish to thank Prof Leendert H.J. Loojienga (JNI Afdeling Pathologie Erasmus MC University Medical Center Rotterdam) for his help in histological sample examination.

\section{References}

1. Hughes IA, Houk C, Ahmed SF, Lee PA, (2006) Consensus statement on management of intersex disorders. Arch Dis Child 91(7): 554-563.

2. Lee PA, Nordenström A, Houk CP, Ahmed SF, Auchus R, et al. (2016) Global DSD Update Consortium: Global Disorders of Sex Development Update since 2006: Perceptions, Approach and Care. Horm Res Paediatr 85(3): 158-180.

3. Ostrer H (2014) Disorders of Sex Development (DSDs): an update. J Clin Endocrinol Metab 99(5): 1503-1509.

4. Luo X, Ikeda Y, Parker KL (1994) A cell-specific nuclear receptor is essential for adrenal and gonadal development and sexual differentiation. Cell 77(4): 481-490.

5. Ferraz-de Souza B, Lin L, Acherman JC (2011) Steroidogenic Factor-1 (SF-1, NR5A1) and human diseases. Mol Cell Endocrinol 336(1-2): 198205.

6. Urs AN, Dammer E, Kelly S, Wang E, Merrill AH, et al. (2007) Steroidogenic factor-1 is a sphingolipid binding protein. Mol Cell Endocrinol 265(266): 174-178.

7. Sablin EP, Blind RD, Krylova IN, Ingraham JG, Cai F, et al. (2009) Structure of SF-1 bound by different phospholipids: evidence for regulatory ligands. Mol Endocrinol 23(1): 25-34.

8. Shen WH, Moore CC, Ikeda Y, Parker KL, Ingraham HA (1994) Nuclear receptor steroidogenic factor 1 regulates the müllerian inhibiting substance gene: a link to the sex determination cascade. Cell 77(5): 651661.

9. Buaas FW, Gardiner JR, Clayton S, Val P, Swain A (2012) In vivo evidence for the crucial role of SF1 in steroid-producing cells of the testis, ovary and adrenal gland. Development 139(24): 4561-4570.

10. Hoivik EA, Lewis AE, Aumo L, Bakke M (2010) Molecular aspects of steroidogenic factor 1 (SF-1). Mol Cell Endocrinol 315(1-2): 27-39.

11. Achermann JC, Ito M, Ito M, Hindmarsh PC, Jameson JL (1999) A mutation in the gene encoding steroidogenic factor- 1 causes XY sex reversal and adrenal failure in humans. Nat Genet 22(2): 125-126.

12. Köhler B, Lin L, Ferraz-de-Souza B, Wieacker P, Heidemann P, et al. (2008) Five novel mutations in steroidogenic factor 1 (SF1, NR5A1) in $46, X Y$ patients with severe underandrogenization but without adrenal insufficiency. Hum Mutat 29(1): 59-64.

13. Coutant R, Mallet D, Lahlou N, Bouhours-Nouet N, Guichet A, et al. (2007) Heterozygous mutation of steroidogenic factor- 1 in $46, \mathrm{XY}$ subjects may mimic partial androgen insensitivity syndrome. J Clin Endocrinol Metab 92(8): 2868-2873.

14. Lourenço D, Brauner R, Lin L, De Perdigo A, Weryha G, et al. (2009) Mutations in NR5A1 associated with ovarian insufficiency. N Engl J Med 360(12): 1200-1210.

15. Camats N, Pandey AV, Fernández-Cancio $M$, Andaluz $P$, Janner $M$, et al. (2012) Ten novel mutations in the NR5A1 gene cause disordered sex development in 46,XY and ovarian insufficiency in 46, XX individuals. J Clin Endocrinol Metab 97(7): E1294-306.

16. Achermann JC, Ozisik G, Ito M, Orun UA, Harmanci K, et al. (2002) Gonadaldetermination and adrenal development are regulated by the orphan nuclear receptor steroidogenic factor-1, in a dose-dependent manner. J Clin Endocrinol Metab 87(4): 1829-1833. 
17. Suntharalingham JP, Buonocore F, Duncan AJ, Achermann JC (2015) DAX1 (NR0B1) and steroidogenic factor-1 (SF-1, NR5A1) in human disease. Best Pract Res Clin Endocrinol Metab 29(4): 607-619.

18. Ahmed SF, Acherman JC, Arlt W, Balen AH, ConwayG, et al. (2011) UK guidance on the initial evaluation of an infant or an adolescent with a suspected disorder of sex development. Clin Endocrinol (Oxf) 75(1): 12 26.

19. Cimador M, Catalano P, Ortolano R, Giuffrè M (2015) The inconspicuous penis in children. Nat Rev Urol 12(4): 205-215.

20. Tantawy S, Lin L, Akkurt I, Borck G, Klingmüller D, Hauffa, et al. (2012) Testosterone production during puberty in two 46,XY patients with disorders of sex development and novel NR5A1 (SF-1) mutations. Eur J Endocrinol 167(1): 125-130.

21. Ciaccio M, Costanzo M, Guercio G, De Dona V, Marino R, et al. (2012) Preserved fertility in a patient with a $46, \mathrm{XY}$ disorder of sex development due to a new heterozygous mutation in the NR5A1/SF-1 gene: evidence of 46,XY and 46,XX gonadal dysgenesis phenotype variability in multiple members of an affected kindred. Horm Res Paediatr 78(2): 119-126.

22. Philibert P, Polak M, Colmenares A, Lortat-Jacob S, Audran F, et al. (2011) Predominant Sertoli cell deficiency in a 46,XY disorders of sex development patient with a new NR5A1/SF-1 mutation transmitted by his unaffected father. Fertil Steril 95(5): 1788.e5-9.

23. Walsh TJ, Dall'Era MA, Croughan MS, Carroll PR, Turek PJ (2007) Prepubertal orchiopexy forcryptorchidism may be associated with lower risk of testicular cancer. J Urol 178(4 Pt1): 1440-1446.

24. Nishina-Uchida N, Fukuzawa R, Numakura C, Suwanai AS, Hasegawa T, et al. (2013) Characteristic Testicular Histology Is Useful for the Identification of NR5A1 Gene Mutations in Prepubertal 46,XY Patients. Horm Res Paediatr 80(2): 119-128.

25. Cools M, Hoebeke P, Wolffenbuttel KP, Stoop H, Hersmus R, et al. (2012) Pubertal androgenization and gonadal histology in two 46,XY adolescents with NR5A1 mutations and predominantly female phenotype at birth. Eur J Endocrinol 166(2): 341-349.

26. El-Khairi R, Achermann JC (2012) Steroidogenic Factor-1 and Human Disease. Semin Reprod Med 30(5): 374-381.

27. Köhler B, Lin L, Mazen I, Cetindag C, Biebermann H, et al. (2009) The spectrum of phenotypes associated with mutations in steroidogenic factor 1 (SF-1, NR5A1, Ad4BP) includes severe penoscrotal hypospadias in 46,XY males without adrenal insufficiency. Eur J Endocrinol 161(2): 237-242. 